\title{
Temperamento en niños de 3 a 7 años con $y$ sin tratamiento psicológico
}

\section{Temperament in children aged 3 to 7 years with and without psychological treatment}

\author{
Luciana Castro $^{1}$ \\ alba.mustaca@uai.edu.ar
}

Alba Elisabeth Mustaca.

albamustaca@gmail.com

\section{Centro del Altos Estudios en Ciencias Humanas y de la Salud (CAECIHS) \\ Facultad de Psicología y Humanidades- Universidad Abierta Interamericana (UAI)}

\section{Resumen}

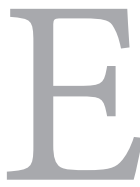

ste trabajo tuvo como objetivos 1) presentar una síntesis del estudio del temperamento infantil que incluyeron distintos enfoques para su estudio y sus relaciones con el género, la edad y problemas de conducta, y 2) evaluar el temperamento infantil en niños de entre 3 y 7 años de una población de Argentina. Se tomó una muestra no aleatoria de 150 niños que fueron evaluados por sus padres o tutores de acuerdo al Cuestionario de Conducta Infantil (Rothbart, Ahadi, Hershey \& Fisher, 2001) en su versión reducida y adaptada al contexto local por Reyna y Brussino (2009). Las niñas puntuaron significativamente más que en los niños en las dimensiones Risa/Sonrisa y Tristeza y no hubo diferencias significativas en función de la edad. Los niños que estaban

\section{Summary}

his study aimed to 1) present a synthesis of the study of child temperament that included the different approaches to study and their relationships with gender, age and behavioral problems; and 2) evaluate the temperament of children between 3 and 7 years of a population of the Buenos aires $y$ Gran Buenos Aires, Argentina. A non-random sample of 150 children was administered the Child Behavior Questionnaire assessed by the parents (Rothbart, Ahadi, Hershey \& Fisher, 2001) in their reduced version and adapted to the local context by Reyna and Brussino (2009). Girls scored significantly more than boys in the Risa / Smile and Sadness dimensions and there were no significant differences as a function

1 Agradecimientos: Investigación parcialmente financiada por la UAI. Se agradece a Mariana Reyna y Silvina Brussino el envío del Cuestionario de Conducta Infantil. 
en tratamiento psicológico puntuaron significativamente más en Atención Focalizada y menos en Ira/Frustración respecto de lo que no estaban.

Palabras clave: Temperamento, niños, tratamiento psicológico of age. Remarkably, children whose parents reported they were on psychological treatment scored significantly more on Focused Care and less on Anger / Frustration than they were not.

Key words: Temperament - Children Population with or without psychological treatment.

\section{Introducción}

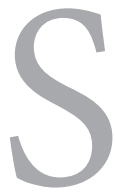

egún Rothbart y Bates (1998) y Rothbart y Derryberry (1981) se considera al temperamento $(\mathrm{T})$ como las diferencias individuales en la reactividad $y$ autorregulación que tienen base constitucional, que se manifiestan en el dominio de la emoción, la actividad y la atención. El componente constitucional hace referencia a las variables biológicas del $\mathrm{T}$, que recibe a la vez influencias de la interacción entre genes y ambiente (Rothbart \& Hwang, 2005). En ese sentido, T y personalidad son conceptos que se confunden ya que, aunque el primero da mayor importancia a los factores biológicos o características que tienen los animales al nacer, el concepto actual agrega la interacción con el ambiente, al igual que el de personalidad. Por otra parte, aunque históricamente el objetivo principal de la investigación sobre la personalidad era identificar aspectos fundamentales en los que los individuos diferían entre sí, más recientemente enfatiza el identificar sus bases biológicas, evolutivas y ontogenéticas por lo cual los conceptos de personalidad y temperamento suelen solaparse entre ellos.
Existen investigaciones del $\mathrm{T}$ más en el campo del desarrollo infantil que en adultos. Una de las razones por lo que es importante estudiar el temperamento infantil (TI) radica en poder detectar las relaciones existentes entre el TI y los problemas de conducta y cuadros psicopatológicos desarrollados en la adolescencia o adultez. Además, existe la necesidad de examinar procesos del desarrollo que pueden estar influenciados por temperamento del niño y cómo tales alteraciones lo colocan en riesgo para actuar de una manera agresiva o antisocial (Frick \& Morris, 2004), o lo contrario, de una manera inhibida, que puede ser foco de acoso o discriminación. Dado que el ambiente puede modular el $\mathrm{T}$, se hace necesario evaluarlos durante el desarrollo para poder implementar intervenciones terapéuticas o educativas, si es necesario, para prevenir y lograr el bienestar futuro de las personas.

Las investigaciones sobre el TI se realizan con una variedad de métodos. Incluyen cuestionarios para padres y maestros, observaciones en laboratorios, en el hogar o en la escuela y medidas fisiológicas mientras los niños realizan diversas pruebas. Todos estos procedimientos tienen sus 


\section{Artículos Empíricos}

ventajas y desventajas. Los cuestionarios tienen la ventaja que son prácticos, económicos y pueden incluir una amplia gama de comportamientos que observan los padres o maestros, de manera de poder explicar la estructura interna del temperamento y evaluarlos a lo largo del desarrollo para establecer componentes constantes o los efectos de intervenciones educacionales o psicológicas. La desventaja es que las respuestas de los cuestionarios pueden tener sesgos culturales, por los afectos o por la personalidad misma de los evaluadores. Sin embargo, son usados con mucha frecuencia por las ventajas que traen a la hora de hacer investigaciones en grandes poblaciones. Es por ello que en la presente investigación se usará un cuestionario efecto de medir el TI.

Los objetivos de este artículo es presentar 1) una revisión sintética de los principales enfoques teóricos en el estudio TI y 2) presentar una investigación cuyo propósito fue evaluar el TI de niños de 3 a 7 años en una población de Buenos Aires y del conurbano de Argentina para extender los resultados obtenidos por Reyna y Brussino (2009) realizados en otras provincias del mismo país. Además, evaluó si existen diferencias en el TI de niños con y sin tratamiento psicológico, algo que no se planteó en las investigaciones anteriores.

Enfoques en el estudio del temperamento infantil.

Si bien la necesidad de clasificar a las personas en función de sus características individuales se remonta a la antigüedad, la investigación moderna sobre el TI comienza alrededor de 1950, aunque a partir de 1980 se convirtió en uno de los temas centrales de la psicología del desarrollo. Según los especialistas (e.g., ElseQuest, Shibley Hyde, Goldsmith, \& Van Hulle, 2006), su estudio se puede clasificar entre un acercamiento inductivo, que comienza con la recolección de hechos sobre conceptos amplios (e.g., Kagan \& Fox, 2006) y otro deductivo, más teórico (e.g., Rothbart \& Bates, 2006), que se aborda teniendo en cuenta hipótesis para luego ponerlas a prueba con trabajos empíricos.

Una investigación pionera sobre el TI fue el Estudio Longitudinal en Nueva York (ELNY, Thomas \& Chess, 1977), claramente inductivo, que comenzó en 1956. Consistió en evaluar a 133 niños de 84 familias de Nueva York, desde los 3 meses de edad hasta la adultez por medio de pruebas fisiológicas y conductuales, observaciones en el hogar y en la escuela y entrevistas a sus padres, parientes y maestros, con el objetivo de hallar regularidades y diferencias en el comportamiento de los niños a lo largo de los años. Determinaron la existencia de clases de niños y dimensiones temperamentales.

Respecto de lo primero, un $40 \%$ fueron catalogados como niños ' 'fáciles ' : generalmente felices, rítmicos" en su funcionamiento biológico e interesados en tener nuevas experiencias; un $10 \%$ son niños ' difíciles ': más irritables y difíciles de complacer, con ritmos biológicos irregulares " más intensos en la expresión de las emociones, y un 15\%, niños ' difíciles de entusiasmar o de adaptación lenta ": afables, aunque se adaptaban lentamente a las personas y situaciones nuevas. Sin embargo, el 35\% de la muestra no se ajustaban en ninguno de estos tres grupos. Por otra parte, los autores reconocieron que los comportamientos que conducen a que un niño sea clasificado como fácil o difícil pueden 


\section{Artículos Empíricos}

variar en función de los valores, actitudes y prácticas parentales y culturales. Por lo tanto, enfatizaron en el concepto de interaccionismo: el desarrollo psicológico no sólo está influenciado por el temperamento del niño, sino también, por las respuestas ambientales a los patrones comportamentales (Thomas \& cols. 1977).

Buss y Plomin (1975) definieron como T a los rasgos que cumplían ciertos criterios basados en la psicología comparada.Losrasgosdetemperamento deben comprobarse en otras especies, en especial en los mamíferos sociales y que tengan una aparición temprana en la ontogénesis. Sostenían que, aunque el $\mathrm{T}$ tiene orígenes en aspectos neurobioquímicos heredables, no permanecerá estático y fijo durante la vida, sino que pueden cambiar por procesos de interacción del genotipo individual con el entorno durante el desarrollo y maduración de la persona. Consideraron que había cuatro rasgos que cumplían esos criterios: 1) la emocionalidad, 2) la actividad, 3) la sociabilidad, y 4) la impulsividad. Sin embargo, esta última luego se descartó debido a que los análisis factoriales mostraron que el rasgo parecía estar compuesto de varios subcomponentes y sólo algunos se replicaron. Finalmente, Buss y Plomin (1984), elaboraron la Escala de Temperamento de Actividad y Sociabilidad (EAS) que mide tres rasgos: (1) emocionalidad: tendencia a activarse fácil e intensamente; actividad: preferencia por un determinado nivel de actividad y velocidad de acción; (3) sociabilidad: tendencia a preferir la presencia de otros más que de permanecer solo o a evitar e inhibirse ante nuevas situaciones sociales.

Goldsmith y Campos (1982) se refieren al $\mathrm{T}$ como las diferencias individuales en las respuestas emocionales, considerándolo como la tendencia a experimentar y expresar emociones, incluyendo sus aspectos reguladores. Para ellos, la emocionalidad se refiere a las distintas emociones primarias tanto positivas como negativas (e.g., la alegría, la tristeza, la ira y el miedo) que se expresan en la intensidad y la duración de la conducta, incluyendo expresiones vocales, faciales y motoras. Junto con Rothbart, Goldsmith desarrolló una amplia batería de evaluaciones de laboratorio, el Lab-TAB y un inventario multidimensional para recolectar índices de TI (Goldsmith \& Rothbart, 1996). Estos instrumentos se diseñaron originalmente para medir cinco componentes emocionales (actividad motora, enojo, miedo, placer / alegría, interés / persistencia), pero luego aparecieron versiones más recientes para evaluar un número mayor de dimensiones (Goldsmith, 2000).

El enfoque actual no sólo considera la emoción, sino también la regulación emocional como un factor del $\mathrm{T}$; siendo éste uno de los constructos más complejos. Se lo definió como los procesos extrínsecos e intrínsecos responsables de monitorear, evaluar y modificar las reacciones emocionales, especialmente en su intensidad y duración (Thompson, 1994). Sin embargo, otros autores argumentaron que la emoción y la regulación emocional pueden considerarse inseparables, ya que ambas ocurren al mismo tiempo, y que las respuestas emocionales dependen de procesos preexistentes de regulación, como la inhibición cortical o las interpretaciones de un evento (Campos, Frankel \& Camras, 2004).

Las relaciones entre la emoción y su regulación también son centrales en el enfoque de Rothbart, aunque pone un mayor énfasis a 


\section{Artículos Empíricos}

los mecanismos atencionales y neurobiológicos. Rothbart (1998), como se afirmó antes, define el $\mathrm{T}$ como las diferencias constitucionales en la reactividad y la autorregulación. La primera se refiere a la excitación biológica, que incluye la activación de los sistemas neuroendocrino, autónomo y afectivo; pueden medirse por el umbral de reacción, la latencia, la intensidad y el tiempo de subida y recuperación ante presencia de determinados estímulos. La autorregulación, en cambio, se refiere a procesos que aumentan, disminuyen, mantienen y reestructuran el patrón de reactividad de una manera anticipada (Rothbart \& Derryberry, 1981). Las conductas relacionadas con la autorregulación incluyen cambios en el enfoque atencional, la evitación, la inhibición y la auto-regulación atencional. Un supuesto importante en este modelo es que ambos comportamientos, los de reactivación y de autorregulación, están íntimamente ligados a diferentes procesos neurobiológicos.

Rothbart y sus colegas identificaron tres dimensiones temperamentales, cada una de las cuales incluye una serie de subdimensiones: (a) extraversión, incluye la anticipación positiva, nivel de actividad y búsqueda de sensaciones; (b) afectividad negativa, incluye el miedo, la irafrustración y el malestar social; y (c) el control eficaz o control esforzado (CE), definido como la capacidad de inhibir una respuesta dominante a favor de otra subdominante en función al logro de una meta futura; incluye el control inhibitorio (CI), la atención focalizada (AF) y la sensibilidad perceptiva (Rothbart \& Bates, 2006). El CE se relaciona con la persistencia, surge alrededor del primer año de vida y aumenta entre los 2 y 3 años. Estas dimensiones están vinculadas a áreas cerebrales y neurotransmisores específicos.
Por ejemplo, la red de atención ejecutiva está relacionada con la corteza cingulada anterior, los ganglios basales y la corteza prefrontal lateral. Una evidencia preliminar sugiere que la neuromodulación de este sistema puede incluir la dopamina (Posner \& Rothbart, 2007).

El enfoque de Kagan y Fox (2006) es inductivo. Kagan, Reznick y Snidman (1988) estudiaron la inhibición conductual y su contrapartida, un estilo desinhibido, basado en una investigación longitudinal de las respuestas conductuales y fisiológicas desde la infancia temprana. Un primer estudio reveló que los niños de 2 años que mostraban una evitación consistente o angustia ante personas, procedimientos y situaciones desconocidas, mantuvieron esas tendencias hasta los 7 años de edad, asociado con un mayor tono del sistema simpático. A la vez, los niños de 2 años que mostraban una mínima evitación o angustia en las mismas situaciones, también tendieron a preservar estas disposiciones conductuales a los 7 años y correlacionaban con un mayor tono del sistema parasimpático. En un segundo estudio examinaron a lactantes en el laboratorio a partir de los 4 meses de edad, y el espectro de medidas fisiológicas se amplió considerablemente en la suposición de que las formas tempranas de la reactividad a la novedad estarían vinculadas a un umbral diferencial de excitabilidad en la amígdala. Kagan y cols. (1988) se centraron en la actividad motora y el llanto como marcadores potenciales de la hiperreactividad de la amígdala. Informaron que alrededor del $20 \%$ de los bebés sanos de 4 meses de edad mostraron una actividad motora frecuente e intensa, y llanto ante la aparición inesperada de estímulos visuales, auditivos u olfativos poco familiares, a los que llamaron niños de "alta reactividad"; y alrededor 


\section{Artículos Empíricos}

del 40\% reaccionaron con una actividad motora y de llanto mínimos ante los mismos eventos y se los llamó de "baja reactividad". En comparación con los bebés de baja reactividad, los de "alta reactividad" tuvieron 3 veces más probabilidades de desarrollar síntomas de ansiedad a los 7 años. Además, los niños con alta reactividad en la adolescencia informaron en una entrevista, episodios más frecuentes de tristeza y mostraron cambios en la frecuencia cardíaca, sudoración de las palmas, tensión muscular, rubor facial y dificultad respiratoria, hipertensión arterial, así como ondas EEG y ERP distintas a los 11 y 15 años de edad respecto de los de baja reactividad (Kagan \& Snidman, 2004). Kagan (2008) considera al alto y bajo nivel de reactividad como categorías distintivas producidas por diferentes factores biológicos y no como puntos en un continuo. Además, conjetura que los fenotipos diferentes se deben originar en genomas también distintos. Por último, Kagan y Snidman (2004) reconocen que pueden surgir una gran variedad de personalidades diferentes de un mismo tipo de temperamento, dependiendo del contexto de crianza, tales como la clase social, la cultura, la familia o la época histórica, aunque el $\mathrm{T}$ impone restricciones. Por ejemplo, un niño que nace con baja reactividad puede convertirse en un investigador, un banquero o un criminal, pero será poco probable que se convierta en una persona que exprese una intensa y perdurable ansiedad ante distintas situaciones de su vida.

Temperamento, género, edad y problemas de conducta.

En relación a las diferencias de género, las revisiones bibliográficas informan poca evidencia de diferencias de temperamento en la infancia, con algunas excepciones (e.g., Bates, 1987; Maccoby \& Jacklin, 1974 y Rothbart, 1986).

Maccoby y Jacklin (1974) presentaron una revisión sobre las diferencias de género donde informan que los muchachos son más "volátiles" que las niñas y que las respuestas emocionales negativas en ellas disminuyen más rápidamente con la edad que en los niños. En cuanto al nivel de actividad, los chicos tienden a ser más activos que las niñas, y esta diferencia se observa después del primer año de vida y aumenta con la edad. Este resultado se confirmó en un meta-análisis de la actividad realizado por Eaton y Enns (1986). Sin embargo, hallaron que la diferencia de género en la actividad fue moderada en magnitud ( $d$ $=0,49)$ y se asoció con la edad: la diferencia de género fue menor en los lactantes $(\mathrm{d}=0,29)$ y aumentó en los niños mayores $(\mathrm{d}=0,64)$.

Las diferencias de género en el comportamiento sonriente informaron que las mujeres sonríen más que los hombres $(\mathrm{d}=0,42$, Hall \& Halberstadt 1986). Un meta-análisis replicó este hallazgo en adolescentes y adultos (d = 0,41, La France, Hecht, \& Paluck, 2003), aunque parece ser inespecífica, porque cuando los hombres y mujeres fueron observados, la diferencia de género fue de menor en magnitud $(\mathrm{d}=0,26)$. Además, si los participantes tenían clara conciencia de que estaban siendo observados, la diferencia de género fue mayor $(d=0,46)$ que si no eran conscientes $(d=0,19)$. La magnitud de la diferencia de género también dependía de la cultura y la edad. Sin embargo, otro meta-análisis anterior halló que la diferencia de género en la sonrisa está ausente en los niños, lo que sugiere que se desarrollan más desde la adolescencia (Hall \& cols., 1986). 


\section{Artículos Empíricos}

Otro estudio indicó que en la adolescencia tardía, las niñas muestran más reactividad emocional que los niños (Bradley,Codispoti, Sabatinell \& Lang, 2001). Maccoby y Jacklin (1974) hallaron que, hasta 18 meses, ambos sexos no difieren en trastornos emocionales y en las reacciones de frustración; después de 18 meses de edad, sin embargo, los niños muestran más emociones y explosiones negativas y un aumento en el nivel de actividad en los varones respecto de las mujeres (Eaton \& Enns, 1986).

El grado en que una cultura valora algunos comportamientos puede impulsar a su reforzamiento, castigo o extinción por parte de la sociedad, y modular los rasgos temperamentales. Varios estudios transculturales mostraron diferencias de TI en culturas orientales vs occidentales. Además, el nivel socioeconómico puede afectar el desarrollo del temperamento a través del riesgo a experimentar eventos negativos o estresantes o experiencias de socialización. Por ejemplo, las culturas colectivistas vs. individualistas y la pobreza extrema, aparecieron como moderadores de las diferencias de temperamento entre los sexos (e.g., Ahadi, Rothbart, \& Ye,1993; Kohnstamm, 1989 y Kochanska, Nazan \& Mary, 2007)

Finalmente, Else-Quest, Hyde, Goldsmith y Van Hulle (2006) presentaron un meta-análisis para estimar la magnitud de las diferencias de género en el nivel medio y la variabilidad de 35 dimensiones y 3 factores de temperamento en niños de 3 meses a 13 años. Evaluaron 205 investigaciones. El control esforzado mostró una gran diferencia que favoreció a las niñas, pero un moderado tamaño el efecto en las dimensiones relacionadas con ese factor ( por ejemplo, Control inhibitorio: $\mathrm{d}=-0,41$, sensibilidad perceptiva: $\mathrm{d}=-0,38)$. Los varones mostraron una mayor incidencia de trastornos externalizadores, rebeldía y mayor participación en juegos activos y bruscos; algunas dimensiones relacionadas con esas conductas resultaron significativas (ej, actividad: $\mathrm{d}=0,33$, placer de alta intensidad: $\mathrm{d}=$ 0,30 ), En cambio, la afectividad negativa mostró diferencias de género insignificantes.

En Argentina, Reyna y Brussino (2015), evaluaron las diferencias del TI en función de la edad, el género y el nivel socio-económico en el comportamiento social (habilidades sociales y problemas de conducta), en relación a las dimensiones temperamentales de la atención focalizada (AF) y el control inhibitorio (CI) en 623 niños de 3,5 y 7 siete años. Hallaron que los niños de 3 años mostraron un menor desempeño que el resto de los grupos, pero el desarrollo no fue lineal en todos los procesos. Los niños de 5 años presentaron un desempeño semejante o mejor en comparación con los de 7 años en algunas de las variables. En las dimensiones de TI el estudio reveló efectos principales significativos de edad tanto en $\mathrm{AF}$ como en $\mathrm{CI}$, y de género; aunque la proporción de varianza parcial explicada fue baja, osciló entre 1\% y $6 \%$. Asimismo, resultó significativa la interacción entre edad y género sobre ambas variables. Los niños de 3 años presentaron un nivel menor de AF y CI respecto de los de 5 y 7 años, aunque entre estos últimos no se apreciaron diferencias estadísticamente significativas. Respecto del género, las niñas mostraron puntuaciones más elevadas en CI que los niños, mientras que no hubo diferencias significativas en AF. Análisis a posteriori sobre los efectos de interacción de la edad y el género sobre la AF encontraron que los varones de 3 años mostraban puntuaciones estadísticamente menores 


\section{Artículos Empíricos}

al resto de los grupos, con excepción de las niñas de 3 años. Estas últimas presentaron niveles menores sólo respecto de las niñas de 5 años y los varones de 7, no diferenciándose de los varones de 5 ni de las niñas de 7 años. Mientras que las niñas de 7 años mostraron una puntuación menor de AF respecto de los varones de 7 , entre los varones y niñas de $5 \mathrm{y}$ los varones de 7 años, no se apreciaron diferencias significativas. Con respecto al CI, informaron que los niños de 3 años presentaron las menores puntuaciones en relación con el resto de los grupos, a excepción de los varones de 5 años. Este último grupo, a su vez, presentó niveles menores de CI respecto de los grupos restantes, con excepción de las niñas de7 años. Las niñas de 5 y los niños de 7 años no se diferenciaron entre sí.

RespectodelTIylos cuadros psicopatológicos, algunos autores dividieron los problemas de conducta en internalizados o externalizados. Los problemas de internalización los definieron como alejamiento social, ansiedad, depresión, problemas psicosomáticos, tristeza, baja regulación de la atención y baja impulsividad. La externalización correspondía a niños tendientes al enojo, impulsividad y baja regulación emocional. Dodge, Lochman, Harnish y Bates (1997) y Eisenberg y cols. (2000) hallaron que los niños con problemas externalizados tenían puntajes altos en enojo, frustración y hostilidad (reactividad y emocionalidad negativa) y bajos en persistencia a la tarea.

Clark y Watson (1991) hallaron que la ansiedad y la depresión se relacionan con altos puntajes de emocionalidad negativa. Una posible explicación sobre esta relación es que estos niños pueden tener dificultades en sus relaciones sociales, lo que provoca más retraimiento y propensos al enojo y a la frustración. Por otra parte, Rothbart y Bates (1998) hallaron que la persistencia se relaciona negativamente con síntomas depresivos.

Betancourt Ocampo y Andrade Palos (2008) relacionaron las dimensiones temperamentales y problemas de externalización vs internalización en una muestra de niños mejicanos de una edad media de 11 años. Hallaron que las mujeres obtuvieron puntajes significativamente más bajos en problemas externalizados y más altos en depresión, ansiedad, somáticos, afectivos, en comparación con los varones. En TI, las niñas presentaron puntajes mayores en persistencia y emocionalidad negativa y más bajos en actividad que los varones. Para las dimensiones de adaptabilidad, reactividad negativa $y$ emocionalidad negativa (juegos) no encontraron diferencias de género. Al igual que en los trabajos previos, la persistencia correlacionó negativamente con los problemas externalizados y la depresión-ansiedad y la adaptabilidad correlacionó positivamente con problemas externalizados, somáticos, de pensamiento, afectivos y de ansiedad.

En resumen, el estudio del TI es complejo y existen diversos abordajes. Sin embargo, la mayoría de los investigadores coinciden que los componentes para definir las características temperamentales residen en que deben tener una aparición ontogénica temprana, una estabilidad moderada, manifestaciones biológicas distintivas y que existan en las especies animales, en especial en mamíferos. Todos los investigadores concuerdan si bien el $\mathrm{T}$ impone restricciones comportamentales, pueden regularse por factores ambientales. En el mismo sentido, Rothbart y Bates (2003) afirman que se pueden considerar los siguientes criterios de 


\section{Artículos Empíricos}

inclusión para el TI: 1) Diferencias individuales en el comportamiento pertenecientes a los dominios de afecto, actividad, atención y sensibilidad sensorial, 2) se expresan en la intensidad de la respuesta, latencia o tiempo de reacción, duración, umbrales y tiempos de recuperación; 3) aparición en los primeros años de vida (aparición parcial en la primera infancia y expresión plena antes de la edad preescolar), 4) contraparte en mamíferos, 5) vinculado a mecanismos biológicos (e.g., neuroquímico, neuroanatómico y genético) y 6) resultados conceptualmente coherentes y relativamente duraderos y predictivos de características comportamentales específicas. Por otra parte, la mayoría de los autores coinciden en la existencia de una serie de dimensiones del temperamento que incluyen esos criterios, y que fueron evaluados y encontrados en la mayoría de las investigaciones contemporáneas. La Tabla I resume las dimensiones que tuvieron mayor evidencia y su definición.

Tabla 1

Resumen de los componentes temperamentales más estudiados

Dimensiones Definición

Dimensiones relacionadas

Inhibición del comportamiento / miedo

Irritabilidad /

frustración

Emocionalidad positiva

Nivel de actividad

Atención /

persistencia

Sensibilidad sensorial (más controvertido)
Inhibición de la conducta en respuesta a personas y situación desconocidas.

Conducta agresiva o irritada en respuesta al dolor o a la frustración.

Propensión a experimentar emociones positivas, típicamente asociada con una conducta enfocada (anticipación positiva, investigación).

Frecuencia, velocidad y vigor del movimiento motor grueso y la locomoción; Intolerancia a la Vivacidad, energía inactividad forzada.

Capacidad para la atención focalizada y el control voluntario del comportamiento.

Control esforzado

Sensibilidad a los estímulos aversivos, tales como ruidos fuertes o rasguños, que se capturan en el constructo incomodidad sensorial y la capacidad Defensa sensorial de reaccionar a estímulos sensoriales de bajo valor estimular (umbrales sensoriales). 


\section{Artículos Empíricos}

Respecto de las relaciones entre género y edad existen investigaciones que muestran diferencias en los estilos temperamentales, pero se encuentran una gran variabilidad en los resultados, lo que podría indicar que están muy influidos por condiciones ambientales. En cuanto a los problemas de conducta, los autores que los clasificaron en externalizado e internalizados hallaron algunas correlaciones con el temperamento evaluado en la infancia temprana que podría predecir con alguna probabilidad problemas futuros. Es de notar que no se hallaron trabajos que evaluaran el TI antes y después de intervenciones clínicas o educacionales.

\section{Método}

\section{Participantes:}

Se utilizó una muestra no probabilística, conformada por 150 niños de entre 3 y 7 años de edad (varones $=81$; mujeres $=69$ ), de diferentes niveles socioeconómicos, que residían en la Ciudad Autónoma de Buenos Aires y en Gran Buenos Aires, Argentina. Se excluyeron de la muestra a los menores cuyos padres informaron que tenían Trastorno Generalizado del Desarrollo debido a que los ítems del instrumento no eran apropiados para ese trastorno. La muestra se dividió en dos grupos en función de si estaban o no en tratamiento psicológico o psiquiátrico informado por los padres. El grupo sin tratamiento (ST) estuvo compuesto por 96 niños (64\% de la muestra total) de los cuales el 58\% $(n=56)$ fueron varones y el $42 \%(n=40)$, mujeres. El grupo con tratamiento psicológico (CT) se compuso por 54 niños (36\% de la muestra total) los cuales 25 (46\%) fueron varones y 29 (54\%) mujeres.

\section{Materiales:}

(1) Cuestionario de Conducta Infantil (CCI) en niñosde 3 a 7 años, versión Argentina, validada por Reyna y Brussino (2009) en Mendoza, Argentina, basado en el Cuestionario de Conducta Infantil de Rothbart, Ahadi, Hershey y Fisher (2001). Consta de 35 ítems que los padres o encargados del niño deben contestar en qué medida cada descripción se adecua al comportamiento del niño durante los últimos 6 meses, categorizados en una escala Likert de 1 (no se adecua) a 7 (se adecua totalmente). A mayor puntuación, mayor es la presencia de cada característica. El CCI está dividido en 6 dimensiones. 1) Distracción (D, en la versión original la denominan distraíbilidad, 5 ítems), indica la tendencia a cambiar el foco atencional; 2) Control Inhibitorio (CI, 7 ítems,) mide la capacidad para planificar y suprimir las respuestas de aproximación inapropiadas bajo instrucciones y en situaciones nuevas o inciertas; 3) Atención Focalizada (AF, 4 ítems), indica la capacidad para mantener el enfoque atencional en los canales relacionados con las tareas; 4)Ira/ Frustración (I/F, 8 ítems) evalúa las reacciones de afectividad negativa relacionada con la interrupción de tareas en curso o en el bloqueo de metas; 5) Sonrisa/Risa (R, 6 ítems), evalúa afecto positivo en respuesta a cambios en la intensidad, ritmo, complejidad e incongruencia de los estímulos y 6) Tristeza (T, 5 ítems), evalúa una afectividad negativa y un estado de ánimo y energía disminuidos, relacionado con la exposición al sufrimiento, la decepción y la pérdida de objetos. El análisis de consistencia interna a través del coeficiente alfa de Cronbach realizado en Mendoza con 222 niños de una población general arrojó buenos índices para las escalas de D $(0,77)$, CI $(0,78)$ e I/F $(0,80)$, 
aceptables para $\operatorname{AF}(0,67)$ y $R(0,68)$ y escasa para $\mathrm{T}(0,58)$. La muestra de la presente investigación tuvo una consistencia interna de muy buena a buena, ya que arrojaron un alfa de Cronbach de 0,84 en $\mathrm{D} ; 0,71$ en $\mathrm{CI} ; 0,66$ en $\mathrm{AF}, 0,85$ en I/F; 0,67 en $\mathrm{R}$ y 0,78 en $\mathrm{T}$.

(2) Cuestionario de datos sociodemográficos. Se recabó información sobre la edad y el género del niño, si se encontraba en tratamiento psicológico y quien respondió al cuestionario (ej., padre, madre u otros familiares).

\section{Procedimiento}

Los cuestionarios se administraron de manera personal y la participación fue voluntaria. Se informó previamente acerca de los objetivos del estudio, así como el carácter anónimo y confidencial de la información brindada. Se presentó una breve descripción en la cual figuraba la consigna de cada test y una justificación de los motivos que orientaban la investigación, y un agradecimiento por la colaboración brindada.

Para los análisis de los resultados se usó el programa SPSS versión 23 y se estableció como criterio de significación un alfa de $\mathrm{p}<0.05$.

\section{Resultados}

En principio se realizó la prueba de normalidad usando el estadístico KolmogorovSmirnov. Las variables no se distribuyeron de forma normal $(\mathrm{p}<0,05)$, excepto I/F $(\mathrm{p}<0,20)$, por lo cual para establecer la significación de las relaciones entre las variables se utilizaron pruebas no paramétricas.

La Tabla 2 muestra los datos descriptivos de las dimensiones obtenidos de la muestra total.

Tabla 2

Puntajes del cuestionario de Conducta Infantil en la muestra total

\begin{tabular}{lcccccc}
\hline & $\mathrm{N}$ & $\mathrm{M}$ & $\mathrm{Md}$ & $\mathrm{DT}$ & Mín. & Máx. \\
\hline Atención Focalizada & 150 & 17,14 & 17 & 5,39 & 5 & 28 \\
Distracción & 150 & 20,15 & 20 & 3,46 & 9 & 29 \\
Control Inhibitorio & 150 & 28,82 & 29 & 7,62 & 8 & 49 \\
Ira/frustración & 150 & 32,66 & 33 & 10,22 & 8 & 56 \\
Tristeza & 150 & 18,51 & 19 & 6,80 & 5 & 35 \\
Risa & 150 & 28,98 & 29 & 6,12 & 14 & 42 \\
\hline
\end{tabular}

Nota: $\mathrm{N}=$ número de sujetos; $\mathrm{M}=$ media; $\mathrm{Md}=$ mediana; DT. Desvío típico; Min. Valor mínimo; Máx= valor máximo. 
En función de la edad, la dimensión $\mathrm{Cl}$ aumentó e I/F y T disminuyeron a los 7 años. Sin embargo, la prueba Kruskal-Wallis indica que ninguna dimensión obtuvo diferencias significativas.

La Figura 1 (izquierda) muestra los rangos promedio de las escalas del CCI en función del género. Un análisis con la prueba MannWhitney mostró que sólo mostraron diferencias significativas las dimensiones $R(Z=-4,072, p<$ $0,0001)$ y $\mathrm{T}(Z=-2,202, \mathrm{p}<0,03)$. En ambos casos las niñas puntuaron más alto que los niños.

La Figura 1 (derecha) muestra el rango promedio de las escalas del CCI en función de los niños CT y ST. Se observa que los valores son en general similares. Sin embargo, se hallan diferencias significativas en AF $(Z=-2,64, p<$ $.008)$ e I/F $(Z=-5.51 ; p<.01)$. Llamativamente, los niños CT muestran puntajes menores de esas tres dimensiones. Dado que aplicando un ANOVA de un factor, los resultados fueron iguales al obtenido con la prueba Mann-Whitney, se calculó el tamaño del efecto con un objetivo exploratorio. En AF se obtuvo un $\eta 2=.043$ y en I/F un $\eta 2=0,04$. De acuerdo a la clasificación de Cohen, el tamaño del efecto es pequeña ya que sugiere que la variable independiente asignada (tratamiento psicológico) explicaría alrededor del $4 \%$ de la varianza total.
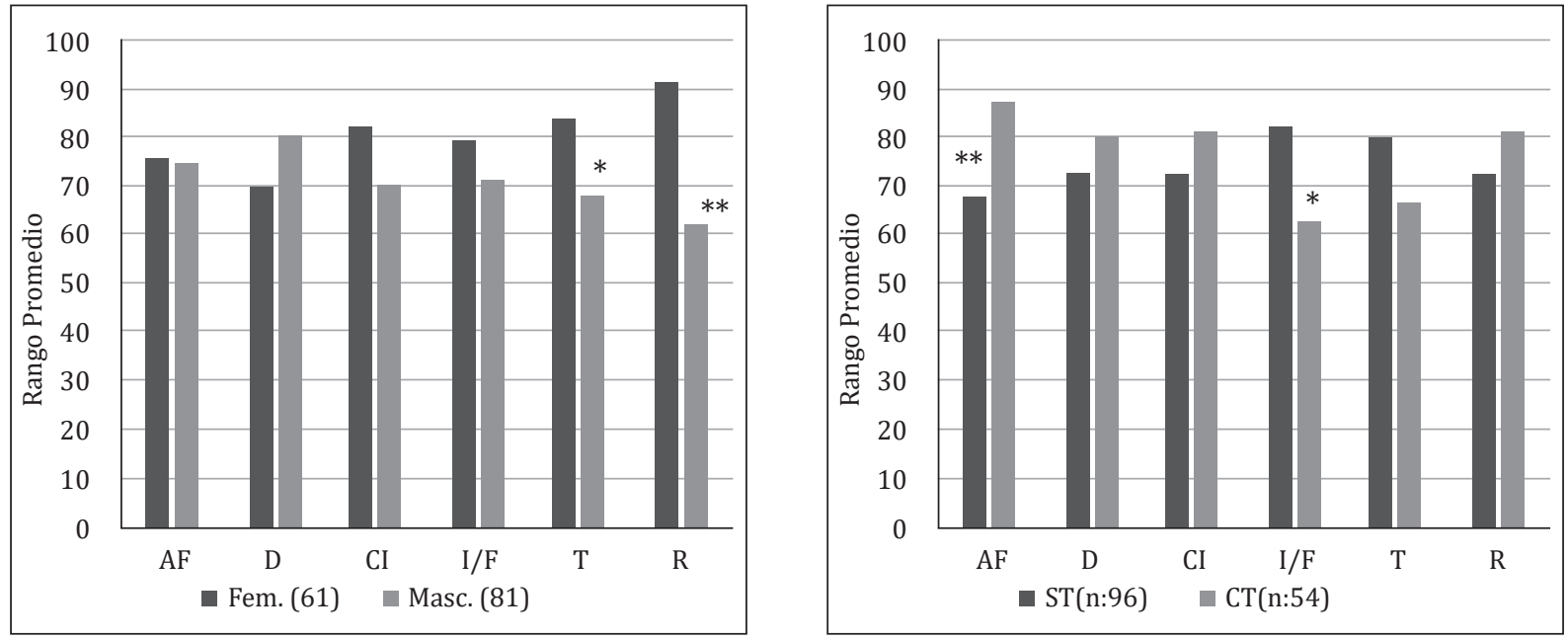

Figura 1. Rango promedio de las escalas del CCI en niños de 3 a 7 años en función del sexo (izquierda) y sin tratamiento (ST) y con tratamiento psicológico (CT, derecha). AF: atención focalizada; D: Distracción; CI: Control Inhibitorio; I/F: Ira/ Frustración; T: Tristeza; R: Sonrisa/Risa. ${ }^{\star}=\mathrm{p}<0,01,{ }^{* *}=\mathrm{p}<0,008$. 
Finalmente, se realizó una correlación múltiple con todas las variables. Las mujeres se categorizaron como 1 y los varones como 2 y 1 si están en tratamiento psicológico y 2 , si no lo están. Los resultados se presentan en la Tabla IV. Se observan correlaciones negativas significativas entre AF con I/F, T y si no están en tratamiento psicológico; entre D con CI, I/F y T; entre CI con I/F y Tristeza; entre T y si no están en tratamiento psicológico y género; y entre $\mathrm{R} y$ género. Las correlaciones significativas positivas se encuentran entre AF con CI, I/F con $\mathrm{T}$ y con tratamiento psicológico; R con CI y T.

Tabla 3

Correlaciones entre las variables

\begin{tabular}{|c|c|c|c|c|c|c|c|c|c|c|}
\hline Dimen. & & $\mathrm{AF}$ & $\mathrm{D}$ & $\mathrm{CI}$ & I & $\mathrm{T}$ & $\mathrm{R}$ & edad & sexo & Trat.psi \\
\hline \multirow[t]{2}{*}{$\mathrm{AF}$} & Co. & 1 & & & & & & & & \\
\hline & Sig. & . & & & & & & & & \\
\hline \multirow[t]{2}{*}{$\mathrm{D}$} & Co. & $-0,007$ & 1 & & & & & & & \\
\hline & Sig. & 0,928 & . & & & & & & & \\
\hline \multirow[t]{2}{*}{$\mathrm{CI}$} & Co. &, $444^{* *}$ &,$- 202 *$ & 1 & & & & & & \\
\hline & Sig. & o & 0,014 & . & & & & & & \\
\hline \multirow[t]{2}{*}{ I } & Co. &,$- 526^{* *}$ &,$- 305^{* *}$ &,$- 191 *$ & 1 & & & & & \\
\hline & Sig. & 0 & 0 & 0,02 & . & & & & & \\
\hline \multirow[t]{2}{*}{$\mathrm{T}$} & Co. &,$- 494^{* *}$ &,$- 340 * *$ & $-0,141$ &, $756^{* *}$ & 1 & & & & \\
\hline & Sig. & o & o & 0,088 & 0 & . & & & & \\
\hline \multirow[t]{2}{*}{$\mathrm{R}$} & Co. & 0,065 & $-0,09$ &, $261 * *$ & 0,095 & ,184* & 1 & & & \\
\hline & Sig. & 0,431 & 0,279 & 0,001 & 0,252 & 0,025 & . & & & \\
\hline \multirow[t]{2}{*}{ edad } & Co. & 0,026 & 0,155 & 0,018 & 0,018 & 0,047 &,$- 166^{*}$ & 1 & & \\
\hline & Sig. & 0,757 & 0,061 & 0,827 & 0,832 & 0,575 & 0,045 & . & & \\
\hline \multirow[t]{2}{*}{ sexo } & Co. & $-0,014$ & 0,117 & $-0,136$ & $-0,086$ &,$- 175^{*}$ &,$- 331 * *$ & 0,03 & 1 & \\
\hline & Sig. & 0,868 & 0,157 & 0,101 & 0,299 & 0,034 & 0 & 0,72 & . & \\
\hline \multirow[t]{2}{*}{ Trat.psi } & Co. &,$- 226 * *$ & $-0,072$ & $-0,107$ &, $203 *$ & 0,151 & $-0,104$ & -0 & 0,124 & 1 \\
\hline & Sig. & 0,006 & 0,383 & 0,199 & 0,013 & 0,067 & 0,211 & 0,96 & 0,134 & . \\
\hline
\end{tabular}

Coeficiente de correlación Rho Pearson. ${ }^{*}=\mathrm{p}<0,01$ (bilateral); ${ }^{*}=\mathrm{p}<0,05$ (bilateral). $\mathrm{N}=147$

AF: atención focalizada; D: Distracción; CI: Control Inhibitorio; I/F: Ira/Frustración; T: Tristeza; R: Sonrisa/Risa. 


\section{Artículos Empíricos}

\section{Discusión}

El temperamento infantil se estudia utilizando diversos enfoques. Sin embargo, hay bastantes coincidencias entre los investigadores respecto de su concepto y de sus dimensiones principales. Todos los autores coinciden que el temperamento incluye las características individuales que son de una aparición ontogénica temprana, tiene una estabilidad moderada a lo largo del desarrollo, manifestaciones biológicas distintivas, que están presentes en una variedad de especies, en especial en mamíferos y que puede ser regulado por el ambiente, aunque impone ciertas restricciones en los patrones de respuesta. En ese sentido, es fundamental evaluar rasgos temperamentales de manera longitudinal y que los puntajes puedan predecir la probabilidad de padecer problemas de conducta o cuadros psicopatológicos en el futuro, con el objeto de poder aplicar intervenciones especiales durante el desarrollo que los eviten o que disminuya su probabilidad de ocurrencia.

En cuanto a los resultados obtenidos en la investigación empírica presentada, se hallaron que las niñas obtuvieron diferencias significativas respecto de los niños en las escalas $\mathrm{T}$ y R. Este resultado coincide parcialmente con otros autores, ya que algunos hallaron que las mujeres superan a los varones en Risa partir de la adolescencia, pero no en Tristeza. Tampoco coincide con lo informado por Reyna y Brussino (2015) y en otros trabajos que hallaron que las niñas presentan una capacidad mayor de AF y $\mathrm{CI}$ en contraste con los varones. El resultado de este estudio sugiere que los padres ven a las niñas más expresivas en las emociones tanto positivas como negativas en relación a los varones, pero sin diferenciarse en AF y en CI.
Respecto a las relaciones entre la edad y las dimensiones temperamentales, según la bibliografía se esperaba que las capacidades de $\mathrm{AF}$ y $\mathrm{Cl}$ se vieran incrementadas en función de la edad de los niños. En esta investigación, si bien se observó un incremento en ambas dimensiones con el aumento de la edad de los niños, las diferencias no alcanzaron significación estadística.

El dato más llamativo está en los puntajes de los niños cuyos padres indicaron que están en tratamiento psicológico. Los niños CT obtuvieron puntuaciones significativamente menores en las AF e I/F que los que los ST, pero, aunque la significación fue alta, el tamaño del efecto fue muy débil. Por ejemplo, Frick y Morris (2004) indicaron que un constructo como "temperamento difícil" un niño puede tener relaciones más específicas con ciertas dimensiones temperamentales. Se esperaría que los niños con patrones de conducta de hiperactividad, agresividad o inadaptación concurrirán con mayor frecuencia a tratamiento psicológico y deberían tener puntuaciones más altas las dimensiones temperamentales que están relacionadas, tales como en I/F y menores en CI y AF y aquellos con una conducta inhibida o con miedos, debería puntuar más en T y CI. Hay varias conjeturas para explicar nuestros resultados. En primer lugar, no tenemos datos sobre las razones por las cuales los niños estaban bajo tratamiento, su diagnóstico ni el tiempo de concurrencia. Tal vez los motivos de consulta y diagnósticos no eran tan severos o hacía varios meses que estaban en tratamiento. Por otra parte, como el CCI lo llenaban padres, podría haber más sesgos perceptuales o un mejor manejo de las respuestas del niño, que 


\section{Artículos Empíricos}

pudieron influir en sus evaluaciones. Además, en los datos de la bibliografía sobre el tema se tomaron simultáneamente inventarios de conductas-problemas y observaciones directas para evaluar las relaciones con las dimensiones del temperamento (ver apartado). Por lo cual se deben realizar futuras investigaciones más controladas para determinar y avalar los resultados de este estudio. Por ejemplo, evaluar el TI, los problemas de conducta y observaciones comportamentales en distintos contextos antes, durante y después de los tratamientos y comparar muestras clínicas que incluyan el diagnóstico,

\section{Referencias}

Ahadi, S. A., Rothbart, M. K., \& Ye, R. (1993). Children's temperament in the US and China: Similarities and differences. European Journal of Personality, 7, 359377.

Bates, J. E. (1987). Temperament in infancy. En J. D. Osofsky (Ed.), Handbook of infant development (pp. 1101-1149). New York: Wiley.

Betancourt, D., Andrade, P. (2008). La influencia del temperamento en problemas internalizados y externalizados en niños. Revista Intercontinental de Psicología y Educación, 10, 29-48.

Bradley, M., Codispoti, M., Sabatinelli, D., \& Lang, P. (2001). Emotion and motivation II: Sex differences in picture processing. Emotion, 1, 300-319. listas de problemas de conducta, tiempo y tipo de tratamiento.

\section{Conclusión}

Finalmente, las correlaciones halladas entre los ítems del cuestionario corroboran lo hallado en estudios anteriores y coincide con las teorías propuestas por Rothbart, M. \& Bates, J. (1998). Por ejemplo, se puede predecir que los niños con altos puntajes en AF también tendrán mayores puntajes en $\mathrm{CI}$ y menores en I/F y en T, mientras que los que tienen puntajes altos en I/F también puntuarán alto en $\mathrm{T}$.

Buss, A., \& Plomin, R. (1975). A temperament theory of personality development. New York: Wiley.

Buss, A., \& Plomin, R. (1984). Temperament: Early developing personality traits. Hillsdale: Erlbaum.

Calkins, S. (2005). Temperament and Its Impact on Child Development: Comments on Rothbart, Kagan, and Eisenberg. Encyclopedia on Early Chilhood Development, 1-6.

Campos, J., Frankel, C., \& Camras, L. (2004). On the nature of emotions regulation. Child Development, 75,377-394.

Chess, S., \& Thomas, A. (1984). Origins and evolution of behavior disorders. New York: Bruncer. 
Clark, L. A. y D. Watson (1991). Tripartite model of anxiety and depresión. Psychometric evidence and taxonomic implications, Journal of Abnormal Psychology, 100, 316336.

Clark, L., \& Watson, C. (1999). Temperament a new paradigm for trait psychology. En A. Lawrence, Handbook of personality (399423). Nueva York: The Guldford Press.

Dodge, K. A., Lochman, J. E., Harnish, J. D., Bates, J. E. y Pettit, G. S. (1997). Reactive and proactive aggression in school children and psychiatrically impaired chronically assaultive youth. Journal of Abnormal Psychology, 106, 37-51.

Eaton, W. O., \& Enns, L. R. (1986). Sex differences in motor activity level. Psychological Bulletin, 100, 19-28.

Eisenberg, N. A., Cumberland, R. A., Spinrad, R. A., Fabes, S. A., Shepard, M., Reiser, B.C., Murphy, S. H., Losoya, I. K. \& Guthrie (2001). The relations of regulation and emotionality to children's externalizing and internalizing problem behavior. Child Development, 72, 1112-1134.

Eisenberg, N., Guthrie, R. A., Fabes, S., Shepard, S., Losoya, B. C., Murphy, S., Jones, R. Poulin, M. \& Reiser, M. (2000). Prediction of elementary school children's externalizing problem behaviors from attentional and behavioral regulation and negative emotionality, Child Development, 71, 1367-1382.
Else-Quest, N. M., Hyde, J. S., Goldsmith, H. H. \& Van Hulle, C. A. (2006). Gender differences in temperament: A metaanalysis. Psychological Bulletin, 6, 33-72.

Frick, P. \& Morris, A. (2004). Temperament and developmental pathways to conduct problems. Journal of clinical child and adolescent psychology, 2, 54-68.

Goldsmith, H. (1996). Studying temperament via construction of the Toddler Behavior Assessment Questionnarire. Child Development, 67, 218-235.

Goldsmith, H. (2000). The Toddler Behavior Assessment Questionnaire Revised. New York: Madison.

Goldsmith, H., \& Campos, J. (1982). Toward a theory of infants temperament. En R. Emde (Comp.). Attachment and affiliative systems (161-193). New York: Plenum Press.

Goldsmith, H. \& Rothbart, M. (1996). Prelocomotor and locomotor Laboratory Temperament Assessment Battery. Ney York: Madison.

Goldsmith, H.; Buss, A.; Plomin, R.; Rotbarth, M.; Thomas, A., \& Chess, S. (1987). Roundtable: What is temperament? Four Approaches. Child Development, 58, 505529.

Hall, J. A. \& Halberstadt, A. G. (1986). Smiling and gazing. En J. S. Hyde \& M. C. Linn (Eds.). The psychology of gender: Advances through meta-analysis (136-158). Baltimore: John Hopkins University Press. 


\section{Artículos Empíricos}

Kagan, J. (2008). The biological contributions to temperaments and emotions. European Journal of Developmental Science, 2, 38-51.

Kagan, J. \& Fox, N. (2006). Biology, culture, and temperament biases. En: E. Eisenberg, Handbook of child psychology Vol 3 (99166). New York: Wiley.

Kagan, J. \& Snidman, N. (2004). The long shadow of temperament. Cambridge: Harvard University Press.

Kagan, J., Reznick, J., \& Snidman, N. (1988). Biological bases on childhood shyness. Science, 240, 167-171.

Kagan, J., Snidman, N., Kahn, V. \& Towsley, S. (2007). The preservation of two infant temperaments through adolescence. Monograph of the Society for Research in Child Development, 287.305.

Kochanska, G., Nazan, A. \& Mary, J. (2007). Children's Fearfulness as a Moderator of Parenting in Early Socialization: Two longitudinal studies. Developmental Psychology, 43, 222-237.

Kohnstamm, G. A. (1989). Temperament in childhood: Cross-cultural and sex differences. En Kohnstamm, G. A., Bates, J. E., \& Rothbart, M. K. (Eds.), Temperament in childhood (483-508). New York: Wiley.

LaFrance, M.; Hecht, M. A. \& Paluck, E. L. (2003). The contingent smile: A meta-analysis of sex differences in smiling. Psychological Bulletin, 129, 305-334.
Lounsbury, M. \& Bates, J. (1982). The cries of infants of differing levels of perceived temperamental difficultness. Child Develompment, 53, 677-686.

Maccoby, E. E., \& Jacklin, C. N. (1974). The psychology of sex differences. Stanford, CA: Stanford University Press.

Posner, M. \& Rothbart, M. (2007). Educating the human brain. Washingtong DC: American Psychological Association.

Reyna, C. \& Brussino, S. (2009). Temperamento infantil: Estudio de fiabilidad de escalas del Cuestionario de Conducta Infantil de Rothbart. XIII Congreso Argentino de Psicología. Córdoba.

Reyna, C. \& Brussino, S. (2015). Diferencias de edad y género en el comportamiento social, temperamento y regulación emocional en niños argentinos. Acta Colombiana de Psicología, 18, 51-64.

Rothbart, M. \& Bates, J. (1998). Temperament. En: N. Eisenberg, Handbook of child psychology, 3, 105-176. New York: Wiley.

Rothbart, M. K. (1986). Longitudinal observation of infant temperament. Developmental Psychology, 22, 356-365.

Rothbart, M. \& Bates, J. (2006). Temperament. En: N. Eisenberg, Handbook of child psychology Vol 3 (99-166). New York: Wiley.

Rothbart, M., \& Derryberry, P. (1981). Development of individual differences in temperament. En E. Lamb, Advances in 


\section{Artículos Empíricos}

developmental psychology, Vol 1, (37-86). Hillsdale: Erlbaum.

Rothbart, M. \& Hwang, J. (2005). Temperament and the development of competence and motivation. En: A. Elliot, \& C. Dweck, Handbook of competence \& motivation (167-184). New York: Guildford Press.

Rothbart, M., Ahadi, S., \& Evans, D. (2000). Temperament and personality: Origins and Outcomes. Journal of Personality and Social Psychology, 78, 122-135.

Rothbart, M., Ahadi, S., Hershey, K. \& Fisher, P. (2001). Investigations of temperament at three to seven years: The children's behavior quetionnaire. Child Development, 75, 1394-1408.

Rothbart, M.,Derryberry,D. \&Hershey, K.(2000). Stability of temperament in childhood. En J. Molfese, (Comp.).Temperament and personality development across the lifespan (88-120). Hillsdale: Erlbaum.

Schawartz, C., Wright, C., Shin, L., Kagan, J. \& Rauch, S. (2003). Inhibited and uninhibited infants "grown up": Adul amygdalar response to novelty. Science, 300, 1952-1953.

Strelau, J. (1983). Temperament-PersonalityActivity. Academic Press.

Thomas, A. \& Chess, S. (1977). Temperament and Development. New York: Brunner.

Thomas, A., Chess, S., Birch, H., Hertzig, M., \& Korn, S. (1963). Behavioral individuality in early childhood. New York: New York University Press.

Thompson, R. (1994). Emotion regulation: A theme in search of definition. Monographs of the Society for Research in Child Development, 59-65. 


\section{Apéndice}

\section{Cuestionario de conducta infantil Adaptación Reyna \& Brusino, 2009.}

A continuación, se presenta un listado de reacciones de los niños ante determinadas situaciones. No existe una forma "correcta" de reaccionar; los niños se diferencian mucho en sus reacciones, son precisamente estas diferencias las que pretendemos captar. Todas las respuestas son confidenciales y anónimas.

Por favor, lea cada descripción y decida EN QUÉ MEDIDA SE ADECUA a cada descripción al comportamiento de su hijo/a durante los últimos 6 meses. Encierre con un círculo el número correspondiente en cada descripción según la siguiente escala:

1. NO se adecua a su hijo o no ha tenido la oportunidad de observarlo.

2. Se adecua POQUÍSIMO a su hijo.

3. Se adecua POCO a su hijo.

4. Se adecua MÁS O MENOS a su hijo.

5. Se adecua MUCHO a su hijo.

6. Se adecua MUCHÍSIMO a su hijo.

7. Se adecua TOTALMENTE a su hijo.

\begin{tabular}{|c|c|c|c|c|c|c|c|}
\hline & 1 & 2 & 3 & 4 & 5 & 6 & 7 \\
\hline $\begin{array}{l}\text { 1. Cuando recoge juguetes o realiza otra tarea, continúa hasta que } \\
\text { termina la actividad. }\end{array}$ & 1 & 2 & 3 & 4 & 5 & 6 & 7 \\
\hline 2. Puede bajar el nivel de su voz cuando se lo piden. & 1 & 2 & 3 & 4 & 5 & 6 & 7 \\
\hline 3. Hace berrinches cuando no consigue lo que quiere. & 1 & 2 & 3 & 4 & 5 & 6 & 7 \\
\hline $\begin{array}{l}\text { 4. Se siente bastante frustrado/a cuando le impiden hacer algo que } \\
\text { quiere hacer. }\end{array}$ & 1 & 2 & 3 & 4 & 5 & 6 & 7 \\
\hline $\begin{array}{l}\text { 5. Llora desconsoladamente cuando uno de sus juguetes favoritos se } \\
\text { pierde o se rompe. }\end{array}$ & 1 & 2 & 3 & 4 & 5 & 6 & 7 \\
\hline $\begin{array}{l}\text { 6. Le cuesta mucho abandonar una actividad cuando le piden que haga } \\
\text { otra cosa. }\end{array}$ & 1 & 2 & 3 & 4 & 5 & 6 & 7 \\
\hline 7. Hace berrinches cuando lo/la critican, aunque lo hagan suavemente. & 1 & 2 & 3 & 4 & 5 & 6 & 7 \\
\hline $\begin{array}{l}\text { 8. Cuando realiza una actividad, le cuesta mantener la atención sobre } \\
\text { ella. }\end{array}$ & 1 & 2 & 3 & 4 & 5 & 6 & 7 \\
\hline 9. Le gustan las historias divertidas y se ríe con ellas. & 1 & 2 & 3 & 4 & 5 & 6 & 7 \\
\hline $\begin{array}{l}\text { 10. Puede esperar a comenzar una nueva actividad si le piden que } \\
\text { espere. }\end{array}$ & 1 & 2 & 3 & 4 & 5 & 6 & 7 \\
\hline 11. Se enoja cuando no encuentra algo con lo que quiere jugar. & 1 & 2 & 3 & 4 & 5 & 6 & 7 \\
\hline
\end{tabular}


12. Le cuesta hacer fila esperando algo.

13. Tiende a ponerse triste si los planes familiares no se realizan.

14. Tiene una actitud pensativa cuando le hablan, como si estuviera en otra parte.

15. Le resulta difícil permanecer sentado/a y callado/a cuando se lo piden (aula, iglesia, cine, etc.).

16. Se frustra con facilidad cuando está cansado/a.

17. Suele tener expresión seria, incluso cuando juega.

18. Necesita completar una actividad antes que le pidan comenzar con otra tarea.

19. Puede seguir instrucciones.

20. Se enoja cuando tiene que comer algo que no le gusta.

21. Se disgusta cuando familiares o amigos queridos se disponen a irse después de una visita.

22. Puede parar fácilmente una actividad cuando le dicen "no".

23. Cambia de una tarea a otra sin terminar ninguna de ellas.

24. Se ríe mucho cuando juega con otros niños.

25. Se enoja fácilmente si encuentra algún problema al realizar una tarea (ej. construir, pintar, vestirse, etc).

26. Sus sentimientos son fácilmente heridos por lo que dicen sus padres.

27. Sonríe o ríe sin motivo cuando juega solo/a.

28. Se distrae fácilmente cuando escucha un cuento.

29. Es capaz de resistir la tentación de hacer algo cuando le dicen que no debe hacerlo.

30. Se pone furioso/a cuando es provocado/a por otros niños.

31. Se pone a llorar cuando le dicen que haga algo que no quiere hacer.

32. Parece seguir su propia dirección, aun cuando le piden hacer algo diferente.

33. Se ríe mucho cuando ve comedias en la televisión o en el cine.

34. Parece no oírme cuando le hablo.

35. Sonríe cuando mira los dibujos de un libro.

\begin{tabular}{|c|c|c|c|c|c|c|}
\hline 1 & 2 & 3 & 4 & 5 & 6 & 7 \\
\hline 1 & 2 & 3 & 4 & 5 & 6 & 7 \\
\hline 1 & 2 & 3 & 4 & 5 & 6 & 7 \\
\hline 1 & 2 & 3 & 4 & 5 & 6 & 7 \\
\hline 1 & 2 & 3 & 4 & 5 & 6 & 7 \\
\hline 1 & 2 & 3 & 4 & 5 & 6 & 7 \\
\hline 1 & 2 & 3 & 4 & 5 & 6 & 7 \\
\hline 1 & 2 & 3 & 4 & 5 & 6 & 7 \\
\hline 1 & 2 & 3 & 4 & 5 & 6 & 7 \\
\hline 1 & 2 & 3 & 4 & 5 & 6 & 7 \\
\hline 1 & 2 & 3 & 4 & 5 & 6 & 7 \\
\hline 1 & 2 & 3 & 4 & 5 & 6 & 7 \\
\hline 1 & 2 & 3 & 4 & 5 & 6 & 7 \\
\hline 1 & 2 & 3 & 4 & 5 & 6 & 7 \\
\hline 1 & 2 & 3 & 4 & 5 & 6 & 7 \\
\hline 1 & 2 & 3 & 4 & 5 & 6 & 7 \\
\hline 1 & 2 & 3 & 4 & 5 & 6 & 7 \\
\hline 1 & 2 & 3 & 4 & 5 & 6 & 7 \\
\hline 1 & 2 & 3 & 4 & 5 & 6 & 7 \\
\hline 1 & 2 & 3 & 4 & 5 & 6 & 7 \\
\hline 1 & 2 & 3 & 4 & 5 & 6 & 7 \\
\hline 1 & 2 & 3 & 4 & 5 & 6 & 7 \\
\hline 1 & 2 & 3 & 4 & 5 & 6 & 7 \\
\hline 1 & 2 & 3 & 4 & 5 & 6 & 7 \\
\hline
\end{tabular}

Edad del niño: Sexo:

Está en tratamiento psicológico o psiquiátrico? Sí No Quién respondió al cuestionario: Madre Padre otro (especificar)

Observaciones: 